\title{
Plumbagin exerts protective effects in nucleus pulposus cells by attenuating hydrogen peroxide-induced oxidative stress, inflammation and apoptosis through NF-kB and Nrf-2
}

\author{
HUI CHU ${ }^{1,2}$, HANG YU ${ }^{1}$, DING REN ${ }^{1}$, KEJUN ZHU $^{1}$ and HONG HUANG ${ }^{1}$ \\ ${ }^{1}$ Department of Orthopedics, The 100th Hospital of Chinese People's Liberation Army, Suzhou, Jiangsu 215007; \\ ${ }^{2}$ Medical College of Soochow University, Suzhou, Jiangsu 215123, P.R. China
}

Received April 20, 2015; Accepted April 1, 2016

DOI: $10.3892 / \mathrm{ijmm} .2016 .2564$

\begin{abstract}
Plumbagin, one of the constituents responsible for the various biological activities of Plumbago zeylanica has been demonstrated to possess antioxidant activity, which may inhibit lipid peroxidation in a dose- and time-dependent manner. In the present study, we aimed to examine the protective effects of plumbagin as well as the underlying mechansim through which plumbagin attenuates hydrogen peroxide $\left(\mathrm{H}_{2} \mathrm{O}_{2}\right)$-induced oxidative stress in nucleus pulposus cells (NPCs). For this purpose, the NPCs were incubated with fresh medium containing $\mathrm{H}_{2} \mathrm{O}_{2}(200 \mu \mathrm{M})$ at $37^{\circ} \mathrm{C}$ in a humidified $5 \% \mathrm{CO}_{2}$ atmosphere for $6 \mathrm{~h}$, and cultured with various concentrations of plumbagin $(0,0.5,1,2,5,10$ and $20 \mu \mathrm{M})$. Treatment with plumbagin significantly increased the viability of the $\mathrm{H}_{2} \mathrm{O}_{2}$-exposed NPCs in a dose-dependent manner. Moreover, plumbagin significantly reduced the generation of reactive oxygen species, lipid peroxidation, as well as the levels of tumor necrosis factor- $\alpha$ (TNF- $\alpha$ ), interleukin (IL)- $1 \beta$ and IL-6 in the $\mathrm{H}_{2} \mathrm{O}_{2}$-exposed NPCs. Glutathione (GSH) content, as well as the activity of catalase (CAT), superoxide dismutase (SOD) and glutathione peroxdiase (GSH-Px) were increased. We found that the administration of plumbagin significantly inhibited the activity of caspase- 9 and -3 , and downregulated $\mathrm{NF}-\kappa \mathrm{B}$ expression and upregulated $\mathrm{Nrf}-2$ expression in the $\mathrm{H}_{2} \mathrm{O}_{2}$-exposed NPCs. Taken together, these findings suggest that plumbagin exerts neuroprotective effects in NPCs by attenuating $\mathrm{H}_{2} \mathrm{O}_{2}$-induced oxidative stress, inflammation and apoptosis through mediating the expression of NF- $\mathrm{NB}$ and Nrf-2.
\end{abstract}

Correspondence to: Dr Hong Huang, Department of Orthopedics, The 100th Hospital of Chinese People's Liberation Army, 4 Canglangting Street, Suzhou, Jiangsu 215007, P.R. China

E-mail: honghuang4xc@163.com

Key words: plumbagin, $\mathrm{H}_{2} \mathrm{O}_{2}$-exposed nucleus pulposus cells, oxidative stress, inflammation, apoptosis

\section{Introduction}

Population aging is a major problem in China and it is also a global issue. Degenerative spinal disorders are caused by pathological changes resulting from lumbar disc degeneration, such as lumbar spondylolisthesis, lumbar spinal stenosis and lumbar disc disease, and have gained increasing attention from researchers (1). Lumbar disc degeneration in the early stages, does not cause pain in the waist or leg; it is only when lumbar disc degeneration has progressed to a particular point that spine-related diseases develop, and pressure on the spinal cord or nerve root results in symptoms including paraplegia, bladder dysfunction, lumbar scoliosis, back pain, sciatica, loss of bilateral or unilateral lower limb muscle strength and paresthesia (2). Approximately $90 \%$ of the worldwide population has suffered from pains in the waist or leg, and some individuals may experience permanent incapacitation from the pain (3). Epidemiological data has also shown that the incidence of lumbar disc degeneration is rising, and it is developing in younger patients (4). A high morbidity as well as a trend towards lumbar disc degeneration in younger patients has gained the attention of experts, and research into the etiology, pathogenesis and treatment of disc degeneration is ongoing (5).

A number of degenerative spinal diseases and secondary lesions caused by lumbar disc degeneration are commonly seen in clinical practice, and the cause and the exact mechanisms responsible for these conditions remains unclear. The excessive apoptosis of disc cells directly leads to a reduction in the number of disc cells, resulting in lumbar disc degeneration (6). Nucleus pulposus cells (NPCs) play an important role in maintaining and repairing the environment within the normal intervertebral disc (7). Thus, the excessive apoptosis of NPCs is a direct cause of lumbar disc degeneration (8). Apoptosis may be triggered by the mitochondrial-dependent and the non-mitochondrial-dependent apoptotic signaling pathways (9). The mitochondrial-dependent pathway may be activated by hydrogen peroxide $\left(\mathrm{H}_{2} \mathrm{O}_{2}\right)$-mediated oxidative stress, leading to apoptosis (10).

Plumbago zeylanica has been used in traditional Chinese medicine, as it possesses various pharmacological activities including anti-inflammatory, antibacterial and antitumor effects, as well as the ability to inhibit glycolysis and to 
stimulate the central nervous system $(3,11)$. Plumbagin (5-hydroxy-2-methyl-1,4-naphthoquinone) is one of the active constituents responsible for the various biological activities of P. zeylanica (chemical structure shown in Fig. 1). Plumbagin belongs to the class of compounds known as napthoquinones, which are phenolic compounds, and a phenolic hydroxyl group with a quinone ring structure is the principal active antioxidant group (12). As the phenolic hydroxyl group is an active hydrogen donor, it provides a hydrogen atom to the peroxide free radical of unsaturated fatty acids, and thereby prevents the formation of new radicals, and interrupts the process of fat oxidation (13). The present study was designed to determine whether plumbagin exerts protective effects against oxidative stress in $\mathrm{H}_{2} \mathrm{O}_{2}$-exposed NPCs as well as to elucidate the underlying mechansim responsible for these effects.

\section{Materials and methods}

Animals and cell culture. Male Sprague-Dawley (SD) rats (8 weeks of age) weighing $200 \pm 30 \mathrm{~g}$ were housed in animal quarters at a temperature of $23 \pm 1^{\circ} \mathrm{C}$ and $60-70 \%$ relative humidity on a $12 \mathrm{~h}$ light/dark cycle $(8: 00 ; 20: 00)$. The experimental study was conducted in accordance with the Guide for the Care and Use of Laboratory Animals prepared by Soochow University (Suzhou, China) and received ethics approval from the Ethics Committee of The 100th Hospital of Chinese People's Liberation Army (Suzhou, China). The $\mathrm{SD}$ rats were injected with an overdose of chloral hydrate, and then the L1-L6 lumbar intervertebral discs were collected from the spinal column. The gel-like nucleus pulposus (NP) tissues were harvested from the discs and immediately placed into $25 \mathrm{~cm}^{2}$ plastic bottles containing Dulbecco's modified Eagle's medium (DMEM)/F-12, and 10\% fetal bovine serum (FBS). The NP tissue samples were cultured for $1-2 \mathrm{~h}$ and then digested with $0.01 \%$ trypsin (Beyotime Institute of Biotechnology, Haimen, China) for $30 \mathrm{~min}$ at room temperature. Following the removal of trypsin, the NP tissue samples were washed twice with phosphate-buffered saline (PBS) and digested with $0.5 \%$ collagenase II (Beyotime Institute of Biotechnology) for $4 \mathrm{~h}$ at room temperature. The NPCs were then filtered through a $200-\mu \mathrm{m}$ mesh strainer and incubated with DMEM/F-12, $10 \%$ FBS, and antibiotics (1\% penicillin/streptomycin) at $37^{\circ} \mathrm{C}$ in a humidified $5 \% \mathrm{CO}_{2}$ atmosphere. After 2 days of incubation, the NPCs were washed with PBS and incubated with fresh medium containing $\mathrm{H}_{2} \mathrm{O}_{2}(200 \mu \mathrm{M})$ at $37^{\circ} \mathrm{C}$ in a humidified $5 \% \mathrm{CO}_{2}$ atmosphere for $6 \mathrm{~h}$.

Cell viability. The NPCs (5,000 cells in $100 \mu \mathrm{l}$ of medium) were seeded in 96-well plates and cultured with plumbagin (Sigma-Aldrich China, Inc., Shanghai, China) at various concentrations $(0,0.5,1,2,5,10$ and $20 \mu \mathrm{M})$ at $37^{\circ} \mathrm{C}$ in a humidified $5 \% \mathrm{CO}_{2}$ atmosphere for $24 \mathrm{~h}$. Subsequently, $10 \mu 1$ 3-(4,5-dimethylthiazol-2-yl)-2,5-diphenyltetrazolium bromide (MTT) was added into each well and the cells were incubated at $37^{\circ} \mathrm{C}$ in a humidified $5 \% \mathrm{CO}_{2}$ atmosphere for $4 \mathrm{~h}$. The culture medium was then removed, and 150-200 $\mu \mathrm{l}$ dimethyl sulfoxide (DMSO) was added into each well and shaken for $20 \mathrm{~min}$. The absorbance was read at $540 \mathrm{~nm}$ using a Labsystems Multiskan MS Plate Reader (Synergy2; BioTek Instruments, Winooski, VT, USA).

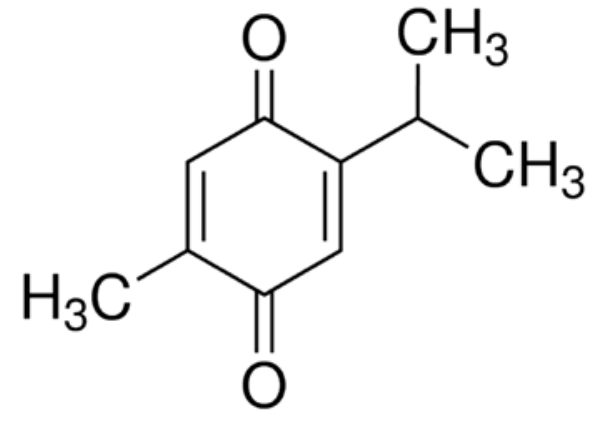

Figure 1. The chemical structure of plumbagin.

Detection of reactive oxygen species (ROS). The NPCs $(5,000$ cells in $100 \mu$ l of medium) were seeded in 96-well plates and cultured with plumbagin $(0,2,5$ and $10 \mu \mathrm{M})$ at $37^{\circ} \mathrm{C}$ in a humidified 5\% $\mathrm{CO}_{2}$ atmosphere for $24 \mathrm{~h}$. ROS activity was examined as previously described by Pi et al (14) using 2',7'-dichlorofluorescein diacetate (DCF-DA; Beyotime Institute of Biotechnology) and measured using a Labsystems Multiskan MS Plate Reader (Synergy2; BioTek Instruments) at an excitation wavelength of $485 \mathrm{~nm}$ and an emission wavelength of $528 \mathrm{~nm}$.

Detection of lipid peroxidation. The NPCs $(5,000$ cells in $100 \mu \mathrm{l}$ of medium) were seeded in 96-well plates and cultured with plumbagin $(0,2,5$ and $10 \mu \mathrm{M})$ at $37^{\circ} \mathrm{C}$ in a humidified $5 \% \mathrm{CO}_{2}$ atmosphere for $24 \mathrm{~h}$. Lipid peroxidation was examined as previously described by Pi et al (14) using a lipid peroxidation kit (Beyotime Institute of Biotechnology) and measured using Labsystems Multiskan MS Plate Reader (Synergy2; BioTek Instruments) at $532 \mathrm{~nm}$ and the results are expressed as $\mathrm{nM}$ thiobarbituric acid reactive substances (TBARS)/mg of protein.

Detection of oxidative stress. The NPCs (5,000 cells in $100 \mu \mathrm{l}$ of medium) were seeded in 96-well plates and cultured with plumbagin $(0,2,5$ and $10 \mu \mathrm{M})$ at $37^{\circ} \mathrm{C}$ in a humidified $5 \% \mathrm{CO}_{2}$ atmosphere for $24 \mathrm{~h}$. The glutathione (GSH) content, as well as the activity of catalase (CAT), superoxide dismutase (SOD) and glutathione peroxdiase (GSH-Px) were detected using commercial kits (Beyotime Institute of Biotechnology) and absornace was measured using a Labsystems Multiskan MS Plate Reader (Synergy2; BioTek Instruments).

Detection of inflammatory cytokines. The NPCs $(5,000$ cells in $100 \mu \mathrm{l}$ of medium) were seeded in 96-well plates and cultured with plumbagin $(0,2,5$ and $10 \mu \mathrm{M})$ at $37^{\circ} \mathrm{C}$ in a humidified $5 \% \mathrm{CO}_{2}$ atmosphere for $24 \mathrm{~h}$. The levels of tumor necrosis factor- $\alpha$ (TNF- $\alpha$ ), interleukin (IL) $-1 \beta$ and IL-6 were detected using commercial kits (Beyotime Institute of Biotechnology) and absorbance was measured using a Labsystems Multiskan MS Plate Reader (Synergy2; BioTek Instruments).

Detection of apoptosis. The NPCs $\left(1 \times 10^{5}\right.$ cells in $100 \mu 1$ of medium) were seeded in 6-well plates and cultured with plumbagin $(0,2,5$ and $10 \mu \mathrm{M})$ at $37^{\circ} \mathrm{C}$ in a humidified $5 \% \mathrm{CO}_{2}$ atmosphere for $24 \mathrm{~h}$. The NPCs were washed twice with cold PBS, and and resuspended in $500 \mu 1$ of binding buffer (BestBio, Inc., Shanghai, China). Thereafter, $5 \mu$ of 


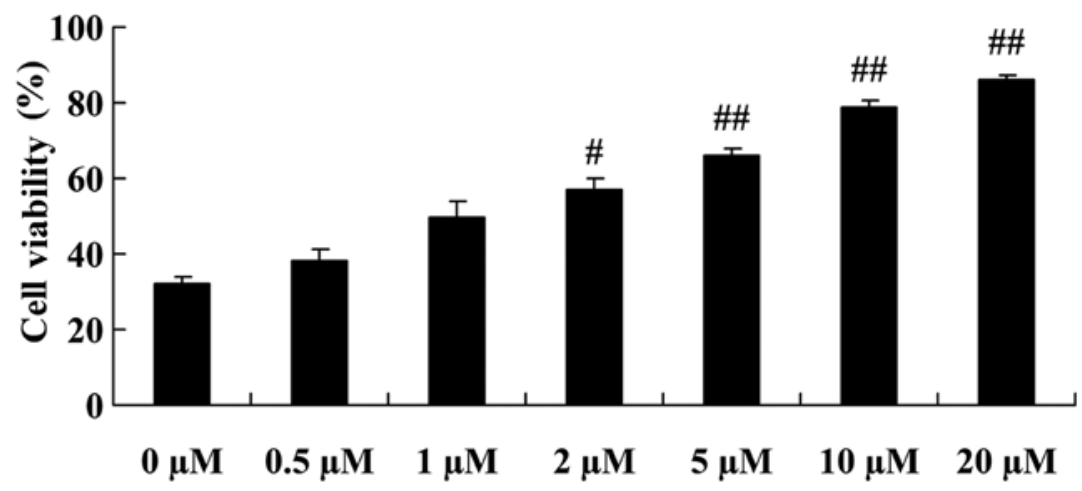

Figure 2. Protective effect of plumbagin increases the cell viability of nucleus pulposus cells (NPCs). ${ }^{\#} \mathrm{p}<0.05$ versus $0 \mu \mathrm{M}$ plumbagin-treated group; ${ }^{\# \#} \mathrm{p}<0.01$ versus $0 \mu \mathrm{M}$ plumbagin-treated group.

Annexin V-FITC was added and incubated for $30 \mathrm{~min}$ at $4^{\circ} \mathrm{C}$ in the dark. Then, $5 \mu \mathrm{l}$ of PI was added in the dark and immediately analyzed on a FACScan flow cytometer (BD Biosciences, San Jose, CA, USA).

Detection of caspase- 9 and -3 activity. The NPCs $\left(1 \times 10^{5}\right.$ cells in $100 \mu 1$ of medium) were seeded in 6-well plates and cultured with plumbagin $(0,2,5$ and $10 \mu \mathrm{M})$ at $37^{\circ} \mathrm{C}$ in a humidified $5 \% \mathrm{CO}_{2}$ atmosphere for $24 \mathrm{~h}$. The NPCs were harvested and resuspended using lysis buffer (Beyotime Institute of Biotechnology) for 30-60 min on ice. The NPCs were harvested by centrifugation $\left(10,000 \mathrm{x} \mathrm{g}\right.$ for $10 \mathrm{~min}$ at $\left.4^{\circ} \mathrm{C}\right)$, and the protein concentration was determined using a bicinchoninic acid protein assay (BCA; KeyGen, Nanjing, China). The activity of caspase- 9 and -3 was detected at a wavelength of $405 \mathrm{~nm}$ using caspase-3 and -9 colorimetric assay kits (Beyotime Institute of Biotechnology) on a FACScan flow cytometer (BD Biosciences).

Western blot analysis of nuclear factor $-\kappa B(N F-\kappa B)$ and nuclear factor erythroid 2-related factor 2 (Nrf-2). The NPCs ( $1 \times 10^{5}$ cells in $100 \mu 1$ of medium) were seeded in 6 -well plates and cultured with plumbagin $(0,2,5$ and $10 \mu \mathrm{M})$ at $37^{\circ} \mathrm{C}$ in a humidified $5 \% \mathrm{CO}_{2}$ atmosphere for $24 \mathrm{~h}$. The NPCs were harvested and resuspended using lysis buffer (Beyotime Institute of Biotechnology) for 30-60 min on ice. The NPCs were harvested by centrifugation $(10,000 \mathrm{x} g$ for $10 \mathrm{~min}$ at $4^{\circ} \mathrm{C}$ ), and the protein concentration was determined using a BCA assay(KeyGEN). Sodium dodecyl sulfate-polyacrylamide gel electrophoresis (SDS-PAGE) was performed with approximately $50 \mu \mathrm{g}$ of sample proteins which were loaded onto $12 \%$ SDS-polyacrylamide gel and then transferred to a nitrocellulose membrane (Bio-Rad Laboratories GmbH, Munich, Germany). The nitrocellulose membrane was blocked with Tris-buffered saline ( $\mathrm{pH}$ 7.4) containing $0.1 \%$ Tween-20 (TBST) and 5\% non-fat milk for $2 \mathrm{~h}$. The membrane was incubated with anti-NF- $\mathrm{BB}$ (3035) and anti-Nrf-2 (12721) (1:1,000; American Diagnostica Inc., Stamford, CT, USA) and anti- $\beta$-actin (A01011, 1:5,000; BestBio, Inc.) overnight at $4^{\circ} \mathrm{C}$. The membrane was washed twice with PBS and incubated with a secondary antibody, horseradish peroxidase-linked anti-rabbit IgG (Santa Cruz Biotechnology, Santa Cruz, CA, USA) for $2 \mathrm{~h}$. The membrane was then incubated with chemiluminescence reagent (ECL Plus Western Blotting Detection system; GE Healthcare, Waukesha, WI, USA). The relative quantity of protein was measured using AlphaEase FC (FluorChem FC2) software (Cell Biosciences Inc., Santa Clara, CA, USA).

Statistical analysis. The results are expressed as the means \pm SEM and were analyzed using the Student's t-test or analysis of variance (ANOVA) as appropriate. A p-value $<0.05$ was considered to indicate a statistically significant difference.

\section{Results}

Protective effect of plumbagin increases the viability of NPCs. We demonstrated that the viability of NPCs was increased by treatment with plumbagin in a dose-dependent manner. Particularly, cell viability was significantly increased following treatment with plumbagin at concentrations of 2 to $20 \mu \mathrm{M}$, compared with that in the $0 \mu \mathrm{M}$ plumbagin-treated group (Fig. 2).

Protective effect of plumbagin decreases the generation of ROS in NPCs. We next examined whether plumbagin exerted a protective effect against the $\mathrm{H}_{2} \mathrm{O}_{2}$-induced generation of ROS in the NPCs. The generation of ROS was highest in the $0 \mu \mathrm{M}$ plumbagin-treated group and there was a significant reduction in ROS levels in the plumbagin-treated groups (2-10 $\mu \mathrm{M})$ (Fig. 3).

Protective effect of plumbagin decreases lipid peroxidation in NPCs. We examined whether plumbagin exerted a protective effect against lipid peroxidation in the $\mathrm{H}_{2} \mathrm{O}_{2}$-exposed NPCs. The results showed that treatment with plumbagin $(2-10 \mu \mathrm{M})$ significantly inhibited $\mathrm{H}_{2} \mathrm{O}_{2}$-induced lipid peroxidation in the NPCs compared with that in the $0 \mu \mathrm{M}$ plumbagin-treated group (Fig. 4).

Protective effect of plumbagin decreases oxidative stress in NPCs. We examined whether plumbagin exerted a protective effect against the $\mathrm{H}_{2} \mathrm{O}_{2}$-induced oxidative stress in the NPCs. As compared with the $0 \mu \mathrm{M}$ plumbagin-treated groups, GSH content as well as the activity of CAT, SOD and GSH-Px in the plumbagin-treated groups $(2-10 \mu \mathrm{M})$ was significantly increased in the $\mathrm{H}_{2} \mathrm{O}_{2}$-exposed NPCs (Fig. 5). 


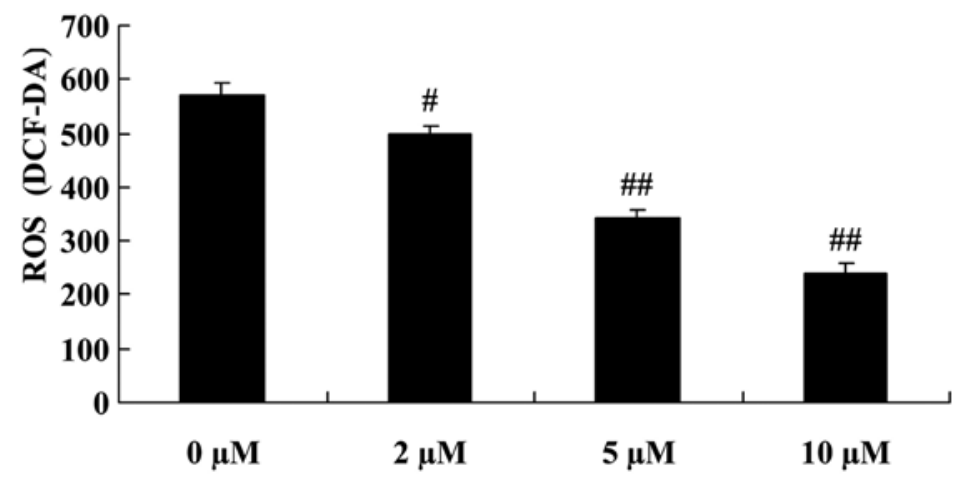

Figure 3. Protective effect of plumbagin decreases the generation of reactive oxygen species (ROS) in nucleus pulposus cells (NPCs). " $\mathrm{p}<0.05$ versus $0 \mu \mathrm{M}$ plumbagin-treated group; ${ }^{\#} \mathrm{p}<0.01$ versus $0 \mu \mathrm{M}$ plumbagin-treated group.

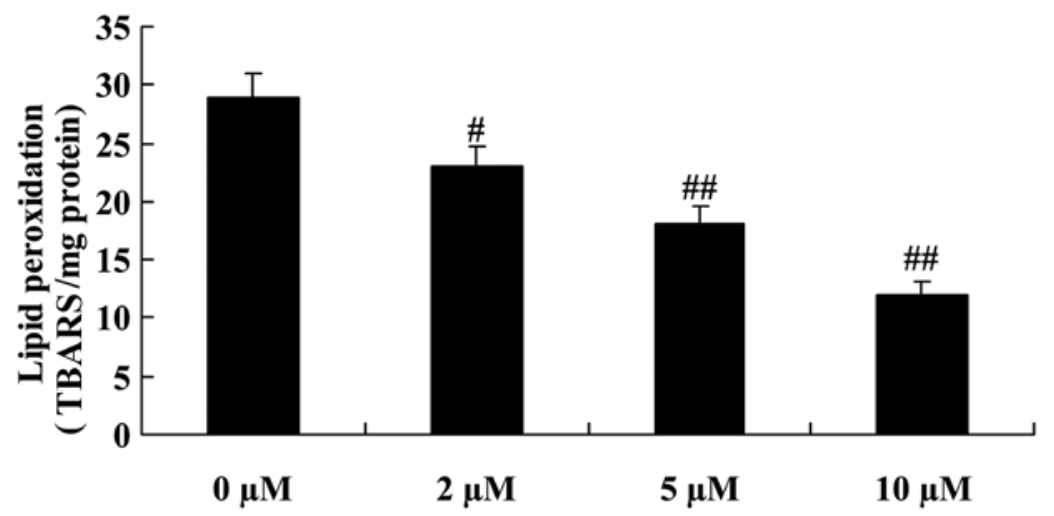

Figure 4. Protective effect of plumbagin decreases lipid peroxidation in nucleus pulposus cells (NPCs). ${ }^{\# p}<0.05$ versus $0 \mu \mathrm{M}$ plumbagin-treated group; ${ }^{\sharp \#} \mathrm{p}<0.01$ versus $0 \mu \mathrm{M}$ plumbagin-treated group.
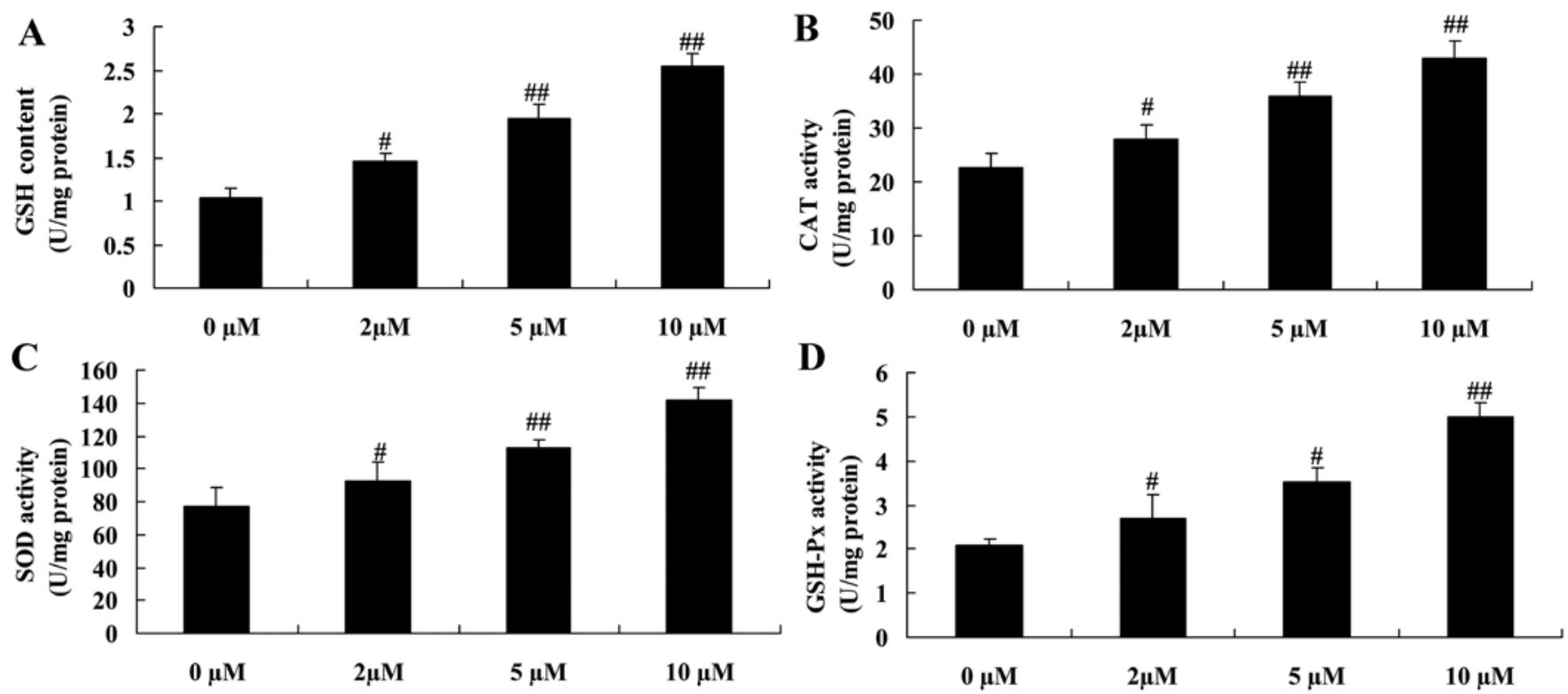

Figure 5. Protective effect of plumbagin decreases oxidative stress in nucleus pulposus cells (NPCs). Plumbagin increased (A) GSH content, as well as the activity of (B) catalase (CAT), (C) superoxide dismutase (SOD) and (D) glutathione peroxdiase (GSH-Px) in the $\mathrm{H}_{2} \mathrm{O}_{2}$-exposed NPCs. " $\mathrm{p}<0.05$ versus $0 \mu \mathrm{M}$ plumbagin-treated group; ${ }^{\# \#} \mathrm{p}<0.01$ versus $0 \mu \mathrm{M}$ plumbagin-treated group.

Protective effect of plumbagin decreases the levels of inflammatory cytokines in NPCs. In order to further explore the protective effects of plumbagin, we examined whether plumbagin decreased the levels of inflammatory cytokines in the $\mathrm{H}_{2} \mathrm{O}_{2}$-exposed NPCs. The administration of plumbagin (2-10 $\mu \mathrm{M})$ significantly decreased the levels of TNF- $\alpha$, IL-1 $\beta$ 

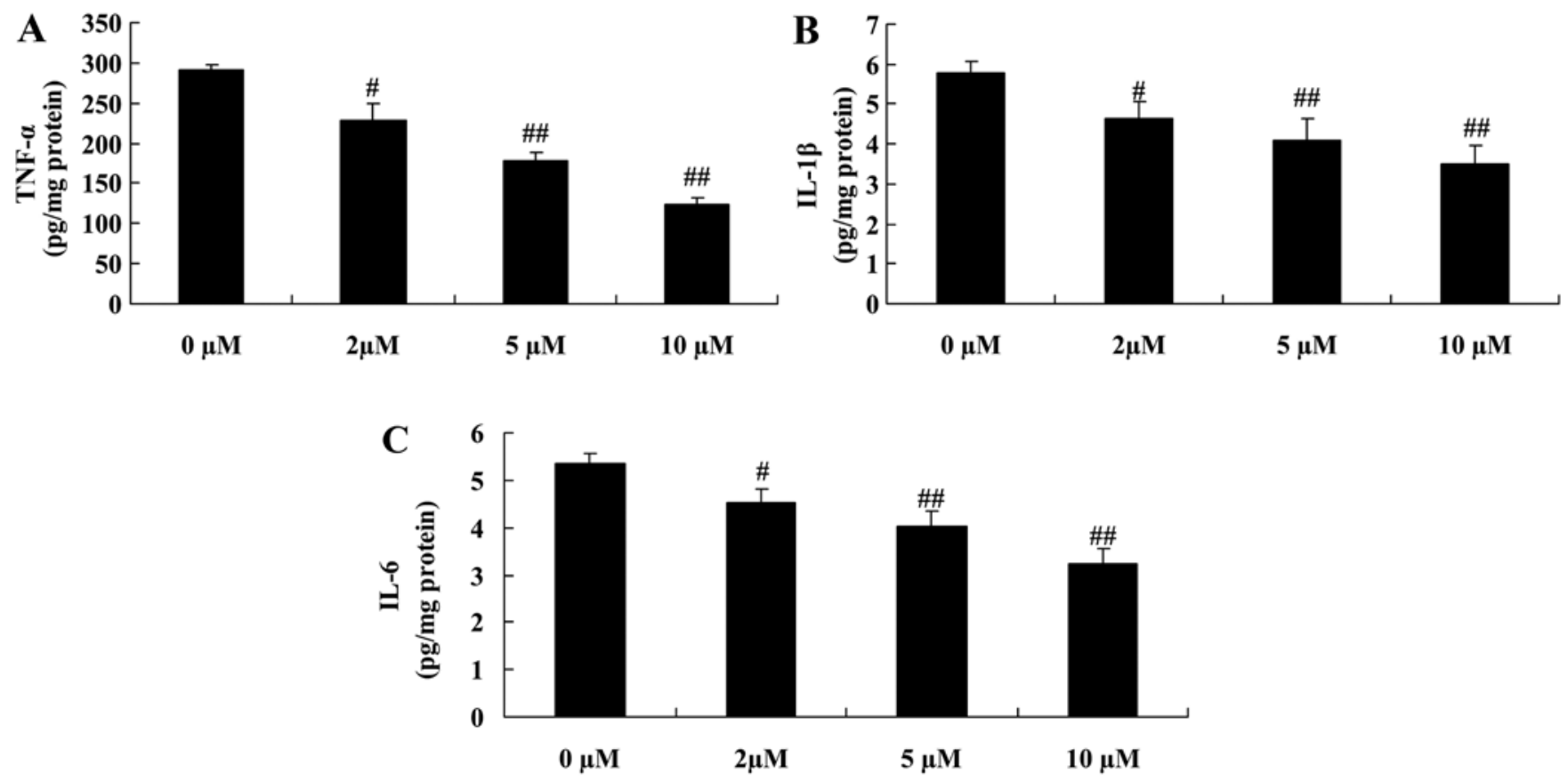

Figure 6. Protective effect of plumbagin decreases the levels of inflammatory cytokines in nucleus pulposus cells (NPCs). Plumbagin decreased the levels of (A) tumor necrosis factor- $\alpha$ (TNF- $\alpha$ ), (B) interleukin-(IL)- $1 \beta$ and (C) IL-6 in the $\mathrm{H}_{2} \mathrm{O}_{2}$-exposed NPCs. ${ }^{*} \mathrm{p}<0.05$ versus $0 \mu \mathrm{M}$ plumbagin-treated group; ${ }^{\#} \mathrm{p}<0.01$ versus $0 \mu \mathrm{M}$ plumbagin-treated group.

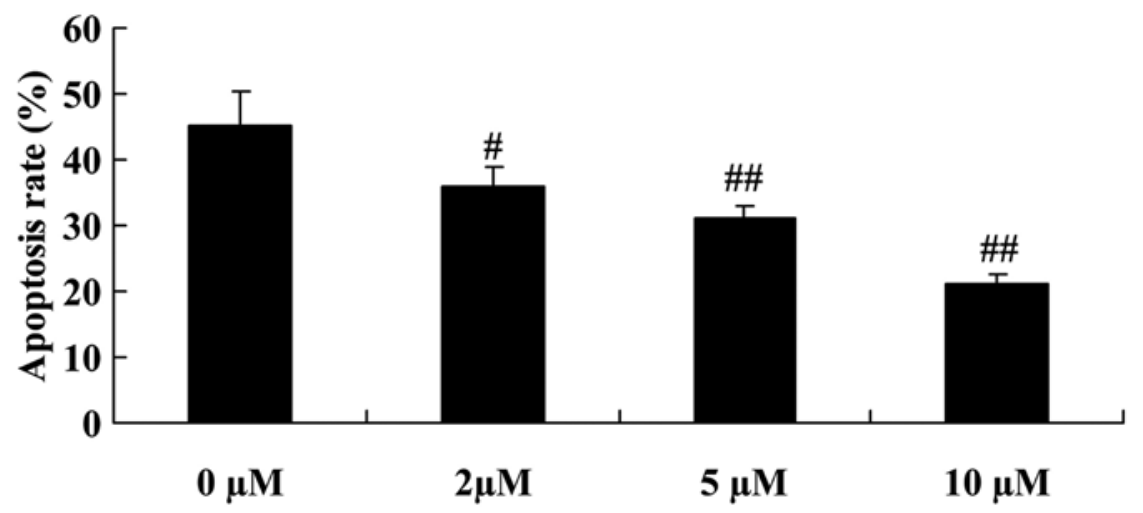

Figure 7. Protective effect of plumbagin decreases the apoptosis of nucleus pulposus cells (NPCs). ${ }^{\#} \mathrm{p}<0.05$ versus $0 \mu \mathrm{M}$ plumbagin-treated group; ${ }^{\#} \mathrm{p}<0.01$ versus $0 \mu \mathrm{M}$ plumbagin-treated group.

and IL-6 in the $\mathrm{H}_{2} \mathrm{O}_{2}$-exposed NPCs compared with those in the $0 \mu \mathrm{M}$ plumbagin-treated groups (Fig. 6).

Protective effect of plumbagin decreases the apoptosis of NPCs. In the next experiment, we examined the antiapoptotic effect of plumbagin in the $\mathrm{H}_{2} \mathrm{O}_{2}$-exposed NPCs As shown in Fig. 7, plumbagin treatment $(2-10 \mu \mathrm{M})$ significantly decreased the cell apoptosis rate in the $\mathrm{H}_{2} \mathrm{O}_{2}$-exposed NPCs, compared with that in the $0 \mu \mathrm{M}$ plumbagin-treated group (Fig. 7).

Protective effect of plumbagin decreases the activity of caspase-9 and -3 in NPCs. In order to further examine the anti-apoptotic effect of plumbagin in the $\mathrm{H}_{2} \mathrm{O}_{2}$-exposed NPCs, the activity of caspase- 9 and -3 was also measured. Caspase- 9 and -3 activity was significantly inhibited in the plumbagin-treated groups $(2-10 \mu \mathrm{M})$, compared with those in the $0 \mu \mathrm{M}$ plumbagin-treated groups (Fig. 8).

Protective effect of plumbagin decreases $N F-\kappa B$ protein expression in NPCs. To explore the anti-inflammatory effect of plumbagin in the $\mathrm{H}_{2} \mathrm{O}_{2}$-exposed NPCs, NF- $\kappa \mathrm{B}$ protein expression was evaluated using western blot analysis. NF- $\kappa \mathrm{B}$ protein expression was significantly suppressed by the administration of plumbagin $(2-10 \mu \mathrm{M})$, compared with that in the $0 \mu \mathrm{M}$ plumbagin-treated group (Fig. 9).

Protective effect of plumbagin increases Nrf-2 protein expression in NPCs. To examine the anti-inflammatory effect of plumbagin in the $\mathrm{H}_{2} \mathrm{O}_{2}$-exposed NPCs, Nrf-2 protein expression was evaluated using western blot analysis. When compared with the $0 \mu \mathrm{M}$ plumbagin-treated group, Nrf-2 

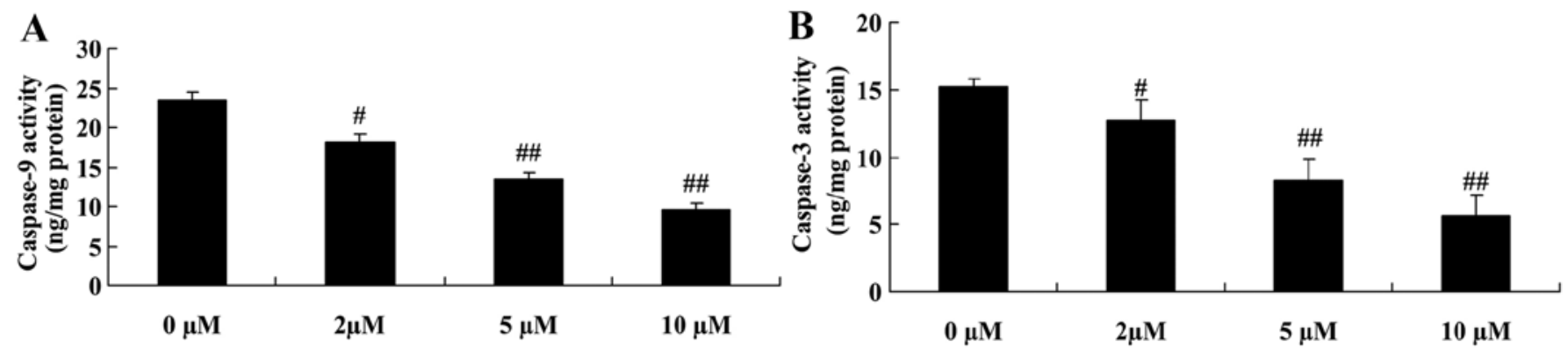

Figure 8. Protective effect of plumbagin decreases the activity of caspase- 9 and -3 in nucleus pulposus cells (NPCs). Plumbagin decreased (A) caspase-9 and (B) caspase-3 activity in the NPCs. $" \mathrm{p}<0.05$ versus $0 \mu \mathrm{M}$ plumbagin-treated group; ${ }^{\# \#} \mathrm{p}<0.01$ versus $0 \mu \mathrm{M}$ plumbagin-treated group.

A

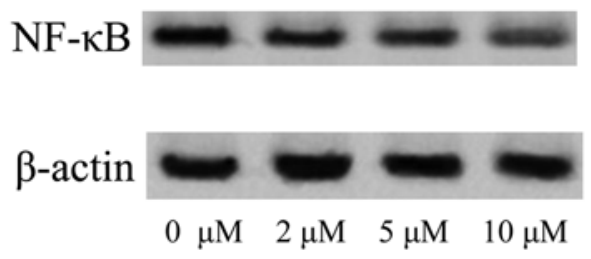

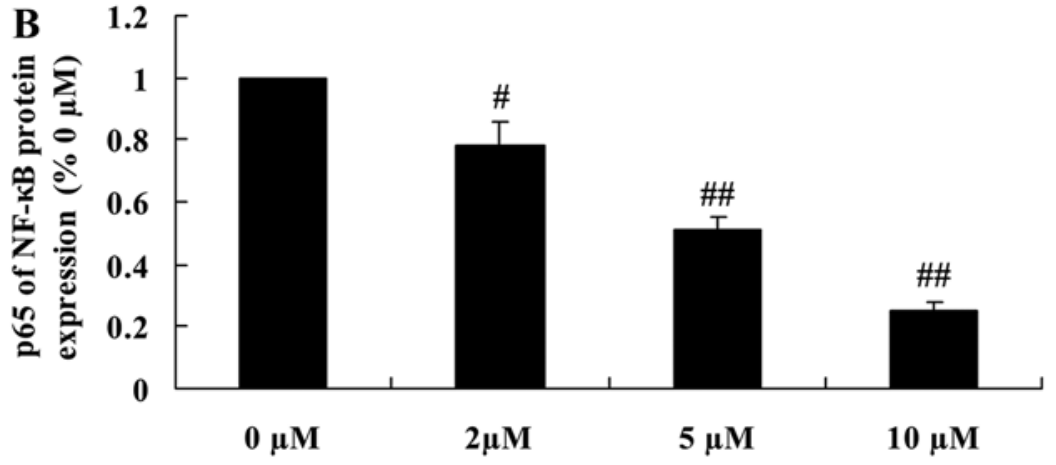

Figure 9. Protective effect of plumbagin decreases nuclear factor- $\mathrm{kB}$ (NF-kB) protein expression in nucleus pulposus cells (NPCs). (A) Plumbagin decreased $\mathrm{NF}-\mathrm{\kappa B}$ protein expression, which was determined using western blot analysis. (B) Quantitative analysis of NF- $\mathrm{kB}$ protein expression in the NPCs. ${ }^{\#} \mathrm{p}<0.05$ versus $0 \mu \mathrm{M}$ plumbagin-treated group; ${ }^{\# \#} \mathrm{p}<0.01$ versus $0 \mu \mathrm{M}$ plumbagin-treated group.

A

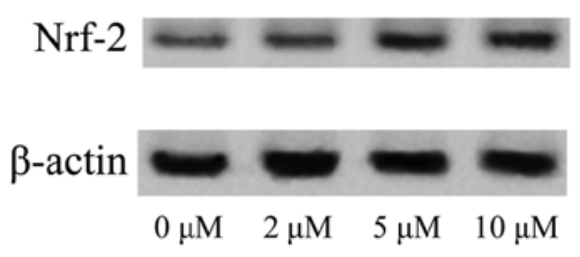

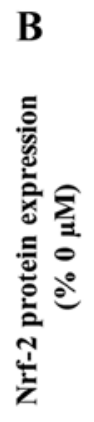

and lifestyle, degenerative spinal disorders caused by lumbar disc degeneration, such as lumbar spondylolisthesis, lumbar spinal stenosis and lumbar disc disease have gained increasing attention $(15,17)$. However, it is proving difficult to treat interveterbral disc degeneration in clinical practice (18). In the present study, we found that treatment with plumbagin significantly increased the viability of $\mathrm{H}_{2} \mathrm{O}_{2}$-exposed NPCs in a dose-dependent manner. Zhang et al suggested that plumbagin protects against spinal cord injury through the suppression of oxidative stress and inflammation through $\mathrm{Nrf}-2$ upregulation in Wistar rats (19). Wang et al reported that plumbagin inhibits of lumbar disc degeneration is rising and it is developing in younger patients (16). With the changes in human longevity

Approximately $90 \%$ of the worldwide population has suffered from pains in the waist or leg to varying extents, and some individuals may experience permanent incapacitation from the pain (15). Epidemiological data also shows that the incidence 
lipopolysaccharide-induced inflammation in RAW 264.7 cells (20).

Oxidative stress refers to the process of tissue damage caused by ROS accumulation in the body or cells as well as the cell toxicity that results from a serious imbalance between free radicals production and the antioxidant defenses when the cells are subjected to harmful stimuli (21). ROS are a class of free radicals which cause oxidative stress, (including $\mathrm{O}_{2}{ }^{-},-\mathrm{OH}, \mathrm{H}_{2} \mathrm{O}_{2}$ and $\mathrm{NO}$, etc.,) and may be produced by mitochondria in human cells, and also induced by environmental stresses (22). Oxidative stress damage in the body is revealed by lipid peroxidation, intracellular protein and enzyme denaturation as well as DNA damage, resulting in abnormal cell function, which ultimately leads to cell death or apoptosis (23). ROS may cause degradation of the extracellular matrix through the inhibition of proteoglycan synthesis and the destruction of extracellular matrix protein structure and protease activity. With increasing age, oxidative stress levels in the body gradually increase as ROS accumulate, causing the destruction of the extracellular matrix in disc tissues and the death or apoptosis of NPCs, thus causing disc degeneration (24). In the present study, treatment with plumbagin significantly reduced the $\mathrm{H}_{2} \mathrm{O}_{2}$-induced generation of ROS as well as lipid peroxidation. GSH content as well as the activity of CAT, SOD and GSH-Px were increased in the NPCs. Zhang et al suggested that plumbagin protects against spinal cord injury through the suppression of oxidative stress,as well as the inhibition of ROS and lipid peroxidation in Wistar rats (19). Checker et al demonstrated that plumbagin inhibits lipopolysaccharide-induced oxidative stress and inflammation (25).

Evidence indicates that the essential pathological changes responsible for lumbar disc degeneration include not only morphological and histological changes, but also a series of changes in biochemical properties (26). Trauma may cause early disc degeneration, which in turn induces the expression of cytokines; the secondary inflammation induces further damage, and moreover increases the severity of lumbar disc degeneration (27). IL-1 $\beta$, IL- 6 and TNF- $\alpha$ are important cytokines, which may be involved in the pathogenesis of lumbar disc degeneration (26). The results of this study showed that the administration of plumbagin significantly decreased the levels of TNF- $\alpha$, IL-1 $\beta$ and IL-6 in the $\mathrm{H}_{2} \mathrm{O}_{2}$-exposed NPCs. A study by Sandur et al indicated that plumbagin abrogated the expression of $\mathrm{NF}-\kappa \mathrm{B}$-regulated gene products which led to the potentiation of apoptosis induced by cytokine and chemotherapeutic agents (28). Luo et al demonstrated that plumbagin inhibits NF- $\kappa \mathrm{B}$ activation and therefore has the potential to be developed as a novel anti-inflammatory agent (29).

Apoptosis may occur under physiological and pathological conditions and circumstances, subject to a variety of factors including caspases. Caspases belong to a family of cysteine hydrolases, which play a central role in apoptosis. Caspase- 3 is an effector of apoptosis (30). NPCs exposed to $\mathrm{H}_{2} \mathrm{O}_{2}$ were used in the present study, and researchers have found that oxidative stress leads to the activation of apoptotic signaling pathways, which include not only the intrinsic apoptotic pathway (such as caspase-9), but also includes the extrinisc apoptotic pathway (caspase-8) as well as the common pathway of both channels (such as caspase-3) (31). This evidence further proves that oxidative stress accelerates the apoptosis of NP cells and causes disc degeneration (32). We found that plumbagin significantly inhibited the activity of caspase- 9 and -3 in the $\mathrm{H}_{2} \mathrm{O}_{2}$-exposed NPCs. Checker et al demonstrated that plumbagin inhibits lipopolysaccharide-induced oxidative stress, inflammation and caspase-3 (25).

The Nrf-2-antioxidant response element (ARE) signaling pathway regulates the encoding of antioxidant proteins through various interactions, and it is the central regulator of cellular antioxidant responses (33). The ARE, which is a cis-acting enhancer sequence of many antioxidant enzyme/protein gene upstream, is one of the defense mechanisms which enables the body to deal with oxidative stress (34). Previous findings have shown that Nrf-2 adjusts the encoding of antioxidant genes by interacting with ARE. Under normal conditions, Nrf-2 is localized in the cytoplasm, combined with the cytoplasmic protein Keap1; when subjected to attack from ROS, Nrf-2 dissociates from Keap1, translocates into the nucleus, binds to Maf protein in order to form a heterodimer, which binds to ARE in the gene to activate targeted gene expression as well as to regulate antioxidant enzyme/protein transcription $(34,35)$. Thus, Nrf-2 is a receptor for oxidative stress, which plays an important role in the protection of cells against oxidative stress and it is one of the principal defense mechanisms induced by exposure to exogenous toxic substances (19). The findings of the present study revealed that plumbagin significantly downregulated $\mathrm{NF}-\kappa \mathrm{B}$ expression and upregulated Nrf-2 expression in the $\mathrm{H}_{2} \mathrm{O}_{2}$-exposed NPCs. Zhang et al suggested that plumbagin protects against spinal cord injury through the suppression of oxidative stress and inflammation through $\mathrm{Nrf}-2$ upregulation in Wistar rats (19).

In conclusion, our findings have demonstrated that plumbagin exerts neuroprotective effects in NPCs by attenuating $\mathrm{H}_{2} \mathrm{O}_{2}$-induced oxidative stress, inflammation and apoptosis. Moreover, we have provided evidence that NF- $\mathrm{B} / \mathrm{Nrf}-2$ are potential targets for plumbagin in intervertebral discs. These findings suggest that plumbagin may be of potential therapeutic value in the treatment of various neurological diseases.

\section{References}

1. Merlini L and Donati U: Auto-immunity and intervertebral disc disease. Ital J Orthop Traumatol 6: 427-432, 1980

2. van den Eerenbeemt KD, Ostelo RW, van Royen BJ, Peul WC and van Tulder MW: Total disc replacement surgery for symptomatic degenerative lumbar disc disease: a systematic review of the literature. Eur Spine J 19: 1262-1280, 2010.

3. Saritha K, Rajesh A, Manjulatha K, Setty $\mathrm{OH}$ and Yenugu S: Mechanism of antibacterial action of the alcoholic extracts of Hemidesmus indicus (L.) R. Br. ex Schult, Leucas aspera (Wild.), Plumbago zeylanica L., and Tridax procumbens (L.) R. Br. ex Schult. Front Microbiol 6: 577, 2015.

4. Castaño-Betancourt MC, Oei L, Rivadeneira F, de Schepper EI, Hofman A, Bierma-Zeinstra S, Pols HA, Uitterlinden AG and Van Meurs JB: Association of lumbar disc degeneration with osteoporotic fractures; the Rotterdam study and meta-analysis from systematic review. Bone 57: 284-289, 2013.

5. Chen S, Liao M, Li J, Peng H and Xiong M: The correlation between microvessel pathological changes of the endplate and degeneration of the intervertebral disc in diabetic rats. Exp Ther Med 5: 711-717, 2013.

6. Liu ZH, Huo JL, Wu ZG, Sun Z, Bai F, Samartzis D, Gantenbein B, Fan SD and Wang HQ: RASSF7 expression and its regulatory roles on apoptosis in human intervertebral disc degeneration. Int J Clin Exp Pathol 8: 16097-16103, 2015. 
7. Sun W, Zhang K, Zhao CQ, Ding W, Yuan JJ, Sun Q, Sun XJ, Xie YZ, Li H and Zhao J: Quantitative T2 mapping to characterize the process of intervertebral disc degeneration in a rabbit model. BMC Musculoskelet Disord 14: 357, 2013.

8. Liu Y, Li JM and Hu YG: Transplantation of gene-modified nucleus pulposus cells reverses rabbit intervertebral disc degeneration. Chin Med J (Engl) 124: 2431-2437, 2011.

9. Wang J, Tang T, Yang H, Yao X, Chen L, Liu W and Li T: The expression of Fas ligand on normal and stabbed-disc cells in a rabbit model of intervertebral disc degeneration: a possible pathogenesis. J Neurosurg Spine 6: 425-430, 2007.

10. Liu ZH, Sun Z, Wang HQ, Ge J, Jiang TS, Chen YF, Ma Y, Wang C, Hu S, Samartzis D and Luo ZJ: FasL expression on human nucleus pulposus cells contributes to the immune privilege of intervertebral disc by interacting with immunocytes. Int J Med Sci 10: 1053-1060, 2013.

11. Dai Y, Hou LF, Chan YP, Cheng L and But PP: Inhibition of immediate allergic reactions by ethanol extract from Plumbago zeylanica stems. Biol Pharm Bull 27: 429-432, 2004.

12. Kumar S, Gautam S and Sharma A: Antimutagenic and antioxidant properties of plumbagin and other naphthoquinones. Mutat Res 755: 30-41, 2013.

13. Chen JW, Ni BB, Zheng XF, Li B, Jiang SD and Jiang LS Hypoxia facilitates the survival of nucleus pulposus cells in serum deprivation by down-regulating excessive autophagy through restricting ROS generation. Int J Biochem Cell Biol 59: $1-10,2015$.

14. Pi H, Xu S, Reiter RJ, Guo P, Zhang L, Li Y, Li M, Cao Z, Tian L, Xie J, et al: SIRT3-SOD2-mROS-dependent autophagy in cadmium-induced hepatotoxicity and salvage by melatonin. Autophagy 11: 1037-1051, 2015.

15. Zhao X, Jiang X and Wang X: Spinal pleomorphic xanthoastrocytoma companied with periventricular tumor. Int J Clin Exp Pathol 8: 1036-1040, 2015.

16. Black RC, Gardner VO, Armstrong GW, O'Neil J and George MS A contoured anterior spinal fixation plate. Clin Orthop Relat Res 227: 135-142, 1988 .

17. Liang QQ, Ding DF, Xi ZJ, Chen Y, Li CG, Liu SF, Lu S, Zhao YJ, Shi Q and Wang YJ: Protective effect of ligustrazine on lumbar intervertebral disc degeneration of rats induced by prolonged upright posture. Evid Based Complement Alternat Med 2014: 508461, 2014.

18. Teichtahl AJ, Urquhart DM, Wang Y, Wluka AE, Heritier S and Cicuttini FM: A Dose-response relationship between severity of disc degeneration and intervertebral disc height in the lumbosacral spine. Arthritis Res Ther 17: 297, 2015.

19. Zhang W, Cheng L, Hou Y, Si M, Zhao YP and Nie L: Plumbagin protects against spinal cord injury-induced oxidative stress and inflammation in Wistar rats through Nrf-2 upregulation. Drug Res (Stuttg) 65: 495-499, 2015.

20. Wang T, Wu F, Jin Z, Zhai Z, Wang Y, Tu B, Yan W and Tang T: Plumbagin inhibits LPS-induced inflammation through the inactivation of the nuclear factor-kappa B and mitogen activated protein kinase signaling pathways in RAW 264.7 cells. Food Chem Toxicol 64: 177-183, 2014

21. $\mathrm{Yu} \mathrm{JH}$ and $\mathrm{Kim} \mathrm{H}$ : Oxidative stress and inflammatory signaling in cerulein pancreatitis. World J Gastroenterol 20: 17324-17329, 2014.

22. Zhang $\mathrm{X}, \mathrm{Ge} \mathrm{Y}, \mathrm{Li} \mathrm{W}$ and $\mathrm{Hu} \mathrm{Y}$ : Diversities of interaction of murine macrophages with three strains of Candida albicans represented by MyD88, CARD9 gene expressions and ROS, IL-10 and TNF- $\alpha$ secretion. Int J Clin Exp Med 7: 5235-5243, 2014.
23. Mohammad NS, Yedluri R, Addepalli P, Gottumukkala SR, Digumarti RR and Kutala VK: Aberrations in one-carbon metabolism induce oxidative DNA damage in sporadic breast cancer. Mol Cell Biochem 349: 159-167, 2011.

24. Cheng YH, Yang SH and Lin FH: Thermosensitive chitosangelatin-glycerol phosphate hydrogel as a controlled release system of ferulic acid for nucleus pulposus regeneration. Biomaterials 32: 6953-6961, 2011.

25. Checker R, Patwardhan RS, Sharma D, Menon J, Thoh M, Sandur SK, Sainis KB and Poduval TB: Plumbagin, a vitamin K3 analogue, abrogates lipopolysaccharide-induced oxidative stress, inflammation and endotoxic shock via NF- $\mathrm{BB}$ suppression. Inflammation 37: 542-554, 2014.

26. Valdes AM, Hassett G, Hart DJ and Spector TD: Radiographic progression of lumbar spine disc degeneration is influenced by variation at inflammatory genes: a candidate SNP association study in the Chingford cohort. Spine 30: 2445-2451, 2005.

27. Omair A, Holden M, Lie BA, Reikeras O and Brox JI: Treatment outcome of chronic low back pain and radiographic lumbar disc degeneration are associated with inflammatory and matrix degrading gene variants: a prospective genetic association study. BMC Musculoskelet Disord 14: 105, 2013.

28. Sandur SK, Ichikawa H, Sethi G, Ahn KS and Aggarwal BB: Plumbagin (5-hydroxy-2-methyl-1,4-naphthoquinone) suppresses NF-kappaB activation and NF-kappaB-regulated gene products through modulation of $\mathrm{p} 65$ and IkappaBalpha kinase activation, leading to potentiation of apoptosis induced by cytokine and chemotherapeutic agents. J Biol Chem 281: 17023-17033, 2006.

29. Luo P, Wong YF, Ge L, Zhang ZF, Liu Y, Liu L and Zhou H: Anti-inflammatory and analgesic effect of plumbagin through inhibition of nuclear factor- $\kappa \mathrm{B}$ activation. J Pharmacol Exp Ther 335: 735-742, 2010

30. Park SE, Shin WT, Park C, Hong SH, Kim GY, Kim SO, Ryu CH, Hong SH and Choi YH: Induction of apoptosis in MDA-MB-231 human breast carcinoma cells with an ethanol extract of Cyperus rotundus L. by activating caspases. Oncol Rep 32: 2461-2470, 2014.

31. Piao CS, Loane DJ, Stoica BA, Li S, Hanscom M, Cabatbat R, Blomgren K and Faden AI: Combined inhibition of cell death induced by apoptosis inducing factor and caspases provides additive neuroprotection in experimental traumatic brain injury. Neurobiol Dis 46: 745-758, 2012.

32. Chen JW, Ni BB, Li B, Yang YH, Jiang SD and Jiang LS: The responses of autophagy and apoptosis to oxidative stress in nucleus pulposus cells: implications for disc degeneration. Cell Physiol Biochem 34: 1175-1189, 2014.

33. Wu Q, Zhang XS, Wang HD, Zhang X, Yu Q, Li W, Zhou ML and Wang XL: Astaxanthin activates nuclear factor erythroid-related factor 2 and the antioxidant responsive element (Nrf2-ARE) pathway in the brain after subarachnoid hemorrhage in rats and attenuates early brain injury. Mar Drugs 12: 6125-6141, 2014.

34. Kaplan O, Meriç M, Acar Z, Kale A, Demircan S, Yılmaz O, Demircan G and Yilmaz Miroğlu Y: The effect of exercise and antioxidant enzyme levels in syndrome $\mathrm{X}$ and coronary slow flow phenomenon: an observational study. Anadolu Kardiyol Derg 13: 641-646, 2013

35. Delgado-Buenrostro NL, Medina-Reyes EI, Lastres-Becker I, Freyre-Fonseca V, Ji Z, Hernández-Pando R, Marquina B, Pedraza-Chaverri J, Espada S, Cuadrado A and Chirino YI: Nrf 2 protects the lung against inflammation induced by titanium dioxide nanoparticles: a positive regulator role of $\mathrm{Nrf} 2$ on cytokine release. Environ Toxicol 30: 782-792, 2015. 